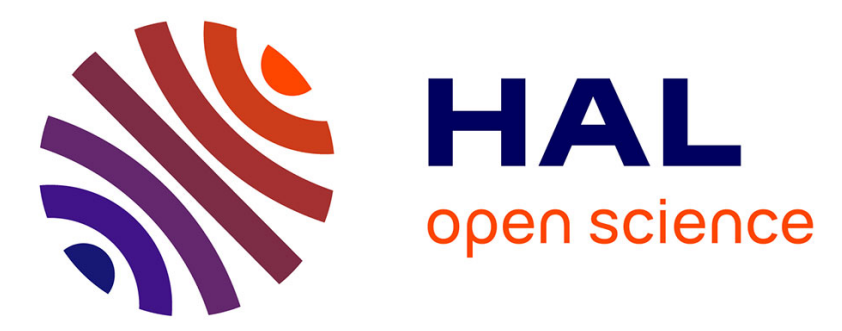

\title{
Identification des enjeux pour les parties prenantes et les acteurs engagés dans la construction d'une marque région : le cas de la marque Auvergne Nouveau Monde
} Corinne Rochette, Cédrine Zumbo-Lebrument, Pierre Féniès

\section{- To cite this version:}

Corinne Rochette, Cédrine Zumbo-Lebrument, Pierre Féniès. Identification des enjeux pour les parties prenantes et les acteurs engagés dans la construction d'une marque région : le cas de la marque Auvergne Nouveau Monde. Gestion et management public [2012-..], 2016, $4 / \mathrm{n}^{\circ} 3$ (1), 10.3917/gmp.043.0091 . hal-01734726

\author{
HAL Id: hal-01734726 \\ https://hal.uca.fr/hal-01734726
}

Submitted on 14 Mar 2018

HAL is a multi-disciplinary open access archive for the deposit and dissemination of scientific research documents, whether they are published or not. The documents may come from teaching and research institutions in France or abroad, or from public or private research centers.
L'archive ouverte pluridisciplinaire HAL, est destinée au dépôt et à la diffusion de documents scientifiques de niveau recherche, publiés ou non, émanant des établissements d'enseignement et de recherche français ou étrangers, des laboratoires publics ou privés. 
IDENTIFICATION DES ENJEUX POUR LES PARTIES PRENANTES ET LES ACTEURS ENGAGÉS DANS LA CONSTRUCTION D'UNE MARQUE RÉGION : LE CAS DE LA MARQUE AUVERGNE NOUVEAU MONDE

\author{
Corinne Rochette, Cédrine Zumbo-Lebrument, Pierre Féniès
}

AIRMAP | «Gestion et management public »

2016/1 Volume $4 / n^{\circ} 3$ | pages 91 à 108

Article disponible en ligne à l'adresse :

https://www.cairn.info/revue-gestion-et-management-public-2016-1-page-91.htm

\title{
Pour citer cet article :
}

Corinne Rochette et al., «Identification des enjeux pour les parties prenantes et les acteurs engagés dans la construction d'une marque région : le cas de la marque Auvergne Nouveau Monde », Gestion et management public 2016/1 (Volume 4 / n ${ }^{\circ}$ 3), p. 91-108.

DOI 10.3917/gmp.043.0091

Distribution électronique Cairn.info pour AIRMAP .

(C) AIRMAP. Tous droits réservés pour tous pays.

La reproduction ou représentation de cet article, notamment par photocopie, n'est autorisée que dans les limites des conditions générales d'utilisation du site ou, le cas échéant, des conditions générales de la licence souscrite par votre établissement. Toute autre reproduction ou représentation, en tout ou partie, sous quelque forme et de quelque manière que ce soit, est interdite sauf accord préalable et écrit de l'éditeur, en dehors des cas prévus par la législation en vigueur en France. Il est précisé que son stockage dans une base de données est également interdit. 


\title{
Identification des enjeux pour les parties prenantes et les acteurs engagés dans la construction d'une marque région : le cas de la marque Auvergne Nouveau Monde
}

\author{
Corinne ROCHETTE ${ }^{1}$, Cédrine ZUMBO-LEBRUMENT ${ }^{2}$, Pierre FÉNIÈS ${ }^{3}$
}

\begin{abstract}
Résumé
La marque territoire est une marque collective partagée impliquant de multiples acteurs au rôle déterminant. La place tenue par les acteurs est explorée à partir d'une grille de lecture combinée issue de la théorie des parties prenantes et de l'analyse stratégique de Crozier et Friedberg. Elle est appliquée au cas de la marque Auvergne Nouveau Monde.
\end{abstract}

Mots clés : Marque territoire, analyse stratégique, Crozier, théorie des parties prenantes

\begin{abstract}
:
A place brand is a shared collective brand involving multiple actors playing a decisive role. The place held by each category of actors is studied with a combined reading grid coming from the stakeholder theory and from the strategic analysis of Crozier and Friedberg. It is applied to the case of the Auvergne Nouveau Monde.
\end{abstract}

Key-words: Place brand, Crozier, strategic analysis of Crozier and Friedberg, stakeholder theory

\footnotetext{
1 Maître de conférences, Ecole Universitaire de Management, IAE d'Auvergne, 11, Boulevard Charles De Gaulle, CS 20273, 63008 ClermontFerrand cedex, téléphone: 04.73.17.77.31, corinne.rochette@udamail.fr

2 Doctorante CIFRE, Agence Régionale de Développement Economique d'Auvergne (ARDE), Université Paris Ouest Nanterre la Défense ; CEROS, cedrine.zumbo-lebrument@orange.fr

3 Professeur des Universités, Université Paris Ouest Nanterre la Défense, CEROS, p.fenies@uparis10.fr
}

\section{Introduction}

Le développement du marketing dans les organisations publiques françaises et de manière plus précise dans les collectivités territoriales s'inscrit dans la lignée du courant du Nouveau Management Public, initié dans les années 1990. Il illustre l'intrusion de pratiques issues de l'univers marchand dans la sphère publique. La création de nombreuses marques territoire ces cinq dernières années est un phénomène important et constitue la manifestation visible de l'adoption d'un marketing de nature stratégique. Les démarches de création de marques concernent des échelons territoriaux variés : des villes, des départements, des régions, des pays de tradition. Le place branding (Anholt, 2010) constitue depuis la fin des années 1990 un courant de recherche actif, mais très centré sur les villes (city branding) (De Chernatony, 1999 ; Kavaratzis, 2004). Les régions en adoptant elles aussi cet instrument qu'est la marque font évoluer leurs pratiques d'une approche essentiellement communicationnelle vers une approche plus stratégique. Le marketing territorial prend alors toute sa pleine mesure.

Le territoire peut être défini comme une étendue de terre administrée par une autorité. II est l'objet d'une appropriation économique, idéologique et politique par les groupes humains qui le constituent et qui cherchent à le valoriser en le rendant plus attractif (Di Méo, 1996) : cette dernière finalité constitue l'objet même du marketing territorial. Un tour des définitions du marketing territorial, données par les chercheurs (Noisette, Vallerugo, 1996; Meyronin, 2009) ou les praticiens (Gollain, 2014 ; Thébault, 2015) permet de constater qu'il s'agit là de l'ambition affichée du 


\section{Identification des enjeux dans la construction d'une marque région Corinne ROCHETTE, Cédrine ZUMBO-LEBRUMENT, Pierre FÉNIÈS}

marketing appliqué au territoire qui s'emploie à faire converger logiques publiques et privées, à coordonner des fonctions du territoire qui peuvent œuvrer en faveur du développement économique et social et de l'attraction d'investisseurs et d'habitants nouveaux. Le marketing territorial vise à différencier le territoire de ceux concurrents, à positionner et valoriser "l'offre territoire ". Le phénomène des marques territoire donne essentiellement lieu à des recherches dans le champ du comportement du consommateur (perception de la marque), du marketing stratégique (positionnement, communication, etc.), mais a peu été étudié sous l'angle organisationnel et processuel (Chanut, Rochette, 2012). La marque territoire en tant que marque collective et partagée est un construit social, elle implique de nombreux acteurs à ce titre elle peut être appréhendée comme une action collective, une action organisée. Elle donne aussi lieu à des organisations particulières. L'approche sociologique mettant l'accent sur le rôle et le comportement des acteurs n'a pas été utilisée pour approcher ce phénomène des marques région alors qu'il constitue à notre sens un cadre d'analyse particulièrement pertinent. Les acteurs embarqués dans le projet sont multiples, leurs objectifs et leurs projets probablement variés, ambigus voire contradictoires avec ceux de l'organisation en charge de la marque, ce qui rend l'agencement organisationnel complexe et le fragilise. Qui sont les acteurs ? Qu'attendentils de la marque région ? Quels sont leurs modes d'action? Pour traiter ces questions, l'adoption d'une approche croziérienne parait particulièrement opportune, aussi nous proposons de mobiliser une lecture stratégique du phénomène à travers les jeux d'acteurs afin d'en évaluer les conditions d'émergence et de maintien de ce construit social et sa dynamique. Dans une première partie, nous présenterons les principes de l'analyse stratégique et ceux de la théorie des parties prenantes. Ce deuxième cadre théorique nous fournit des éléments pour repérer les acteurs et leur comportement dans une organisation (structure portant la marque territoire) marquée par des contours flous. La deuxième partie sera consacrée à la présentation du positionnement de la recherche et du cadre méthodologique avant de présenter et discuter les résultats dans une troisième partie.

\section{La marque territoire expression de l'action collective : vers une lecture croziérienne adaptée du phénomène}

Une première observation, à visée exploratoire, de quelques marques de territoire (trois marques région - Bretagne, Alsace, Auvergne - et une marque urbaine Only Lyon-) réalisée à partir d'une recherche sur les sites internet de ces marques sur l'année 2014 et d'une revue des articles de presse quotidienne publiés sur la période 2009-2014 révèle d'une part une grande fluctuation du nombre et du statut des acteurs impliqués dans la construction et le déploiement de ces marques et d'autre part permet de mettre en évidence le caractère collectif de ces projets («marque collective» «marque co-construite», "marque partagée») et sa structuration autour d'une organisation. En tant qu'objet co-construit la marque territoire implique de nombreux acteurs partageant une préoccupation commune qu'il convient d'étudier de manière plus précise.

\subsection{La marque territoire vue comme une solution collective au déficit d'attractivité}

La création d'une marque territoire constitue une manifestation de l'action collective pouvant être appréhendée à partir du cadre de l'analyse stratégique issu de la sociologie politique, un cadre largement éprouvé, proposé par Crozier et Friedberg (1977).

Le postulat de départ pose l'organisation comme un construit humain constituant une solution crée par des acteurs relativement autonomes qui usent de leurs ressources et de leurs capacités particulières pour résoudre les problèmes posés par l'action collective. Crozier et Friedberg (1977) définissent cette dernière comme une coalition d'hommes contre la nature en vue de résoudre des problèmes. Le problème auquel l'action 
collective s'attaque peut être considéré dans le cas présent comme le déficit d'attractivité de certains territoires dont les collectivités ont la charge et des difficultés à affirmer leurs avantages compétitifs face à une concurrence grandissante entre les territoires (pays, régions, villes). Les objectifs affichés par les organisations en charge des marques

Tableau 1 : Les objectifs des marques territoire (selon les auteurs)

\begin{tabular}{|c|c|}
\hline $\begin{array}{l}\text { Marques } \\
\text { territoire }\end{array}$ & Objectifs / Ambition \\
\hline Bretagne & $\begin{array}{l}\text { "C'est une marque de territoire au service de l'attractivité de la Bretagne. Elle est conçue pour } \\
\text { permettre à la région d'être plus visible et attractive, en enrichissant et en modernisant son } \\
\text { image. " (extrait du site internet officiel de la marque Bretagne) }\end{array}$ \\
\hline Alsace & $\begin{array}{l}\text { "La marque Alsace a pour principal objectif de développer l'attractivité et le rayonnement de } \\
\text { la région en valorisant ses nombreux atouts. Elle vise à mettre en avant une nouvelle image de } \\
\text { l'Alsace, plus complète, plus spécifique et plus attractive. " (extrait du site internet officiel de la } \\
\text { marque Alsace) }\end{array}$ \\
\hline Auvergne & $\begin{array}{l}\text { "L'idée est de rassembler tous les acteurs concernés par l'attractivité de l'Auvergne pour } \\
\text { animer une réflexion concertée sur la stratégie de renforcement de l'attractivité de } \\
\text { l'Auvergne. Unis sous la marque territoriale partagée Auvergne Nouveau Monde - pour une } \\
\text { prise de parole "Auvergne " plus cohérente, lisible et visible - les acteurs d'Auvergne sont } \\
\text { appelés à se mobiliser pour mener ensemble des actions collectives valorisant notre belle } \\
\text { région. L'objectif est de renforcer l'attractivité de la région à travers la marque Auvergne } \\
\text { Nouveau Monde " (extrait du site internet officiel de la marque Auvergne Nouveau Monde) }\end{array}$ \\
\hline OnlyLyon & $\begin{array}{l}\text { "Aujourd'hui, la compétition mondiale entre les villes se joue sur la puissance des } \\
\text { équipements, le poids démographique, ou encore la concentration des fonctions stratégiques, } \\
\text { mais aussi sur la capacité à être repérée par les décideurs et les investisseurs. Grâce à sa } \\
\text { stratégie marketing portée par le label "ONLYLYON", l'agglomération lyonnaise est entrée dans } \\
\text { la course et franchit une nouvelle étape dans sa notoriété européenne et internationale. Son } \\
\text { ambition: devenir l'une des toutes premières métropoles européennes de référence." } \\
\text { (extrait du site internet officiel de la marque ONLY LYON) }\end{array}$ \\
\hline
\end{tabular}

La recherche de solutions visant à renforcer l'attractivité passe par la mise en place de politiques publiques destinées à agir sur les facteurs d'attractivité classiques (infrastructures, cadre de vie, qualité de la main d'œuvre - formation -, pôles de compétitivité, politique culturelle...) (Hatem, 2004 ; Proulx, Tremblay, 2006 ; Thiard, 2007), mais, de façon plus originale, implique aussi la construction et la communication d'une image forte, différenciante et séduisante (Houllier-Guibert, 2009 ; 2011) qui s'incarne depuis le début des années 2000 dans une marque. Le développement de marque partagée s'inscrit dans cette dimension, elle requiert une coopération qui constitue un moyen de réaliser des objectifs communs: renforcer l'attractivité pour différents acteurs publics (collectivités, universités, structures territoire (tableau 1) attestent de la prégnance de la question de l'attractivité qui constitue par ailleurs un objet de recherche investi par les praticiens et chercheurs ces dernières années (Crouch, Ritchie, 1999; Meyronin, 2009; Musson, 2012 ; Baygert, 2014 ; Chamard, 2014).

en charge du tourisme, agence de développement économique...) et privés (entreprises, $\mathrm{CCl}, . .$. ). La marque territoire est vue comme un processus discontinu, coopératif et créatif (Chanut, Rochette, 2012), et constitue un mode d'organisation particulier au sens de Crozier. Cependant, en tant que solution construite, elle pose des difficultés dont la première est celle de la coopération. En effet, construire une marque partagée, c'est pour les acteurs coopérer, car il faut un minimum d'intégration des comportements des groupes et des individus ; or, chaque acteur tend à développer des stratégies à la fois collectives et individuelles avouées ou cachées qui ne sont pas nécessairement compatibles. II s'avère donc délicat d'appréhender la réalité et le fonctionnement des organisations portant les 
marques territoire. Ces aspects ont d'ailleurs été très peu traités (Eshuis, Klijn, Braun, 2013) par les chercheurs en gestion ou ont été abordés de manière succincte, ceux-ci s'étant plus attachés à étudier la transférabilité des pratiques marketing au territoire (Texier, Valla, 1992 ; Noisette, Vallerugo, 1996 ; Girard, 1999), les marques crées et leur rayonnement (Dumont, 2013; Michelet, Giraut, 2014) plus que les acteurs engagés, leurs motivations et leurs modes d'action. Le cadre de l'analyse stratégique proposée par Crozier et Friedberg (1977) peut être mobilisé pour étudier une organisation dont la diversité des acteurs impliqués (issus de cultures organisationnelles publiques et privées) et la grande variété de leurs motivations en font un objet particulièrement complexe.

\subsection{L'analyse stratégique de Crozier et Friedberg}

Pour comprendre le fonctionnement d'une organisation, il convient de s'intéresser aux acteurs et au système (aux relations entre des éléments interdépendants). Pour ce faire, Crozier et Friedberg (1977) proposent d'adopter un double raisonnement: I'un stratégique centré sur l'acteur avec sa stratégie " égoïste » et l'autre systémique est consacré à l'analyse du système et de sa cohérence finalisée. Cette double analyse acteurs-système permet selon les auteurs de comprendre les articulations entre les jeux d'acteurs et le système d'action concret (SAC) et ainsi de rendre compte à la fois de la structuration du champ d'action et de la contribution du jeu des acteurs à cette structuration. Elle permet de dégager les mécanismes et processus de production de l'ordre par lequel les acteurs structurent leur coopération, gèrent leur interdépendance et institutionnalisent leur espace d'action. Les auteurs définissent le SAC comme étant « un ensemble humain structuré qui coordonne les actions de ses participants par des mécanismes de jeux relativement stables et qui maintiennent sa structure, c'est-à-dire la stabilité de ces jeux et les rapports entre ceux- ci par des mécanismes de régulation qui constituent d'autres jeux " (Crozier, Friedberg, 1977, p. 246). Dans le SAC, les processus d'interaction sont régulés par des règles du jeu régissant les dépendances mutuelles des acteurs (Friedberg, 1993). Ces règles permettent de résoudre les problèmes de l'organisation et conduisent à des alliances qui incarnent l'engagement et le soutien dont les acteurs peuvent bénéficier.

Les récentes recherches montrent que l'organisation est un espace d'action collective de moins en moins figé, dont le caractère construit et artificiel, temporaire et fragile apparait de plus en plus clairement dans nos sociétés (Friedberg, 1993) comme en attestent les organisations aux frontières mouvantes dont le fonctionnement apparait de plus en plus désordonné ${ }^{4}$. L'acteur en tant qu'élément central de l'organisation constitue le premier élément d'étude de l'organisation et permet d'en comprendre la nature. Pour Crozier et Friedberg, les acteurs présentent plusieurs caractéristiques; ils disposent de buts et d'objectifs propres rarement clairs, d'une liberté d'interprétation de leur rôle, et d'une rationalité limitée provenant de connaissances et d'informations imparfaites, mais aussi de la disposition à voir l'organisation sous l'angle de leurs objectifs. ${ }^{5}$ Leurs situations d'action sont plus déterminées par les opportunités offertes que par leurs aspirations ou leurs besoins, ainsi leurs actes individuels sont orientés par le contexte d'action. Partant du principe que les acteurs poursuivent des objectifs propres qui ne sont pas nécessairement compatibles avec ceux de l'organisation, ils adoptent des comportements pour préserver leurs intérêts, règlent leurs coopérations en mettant en place des moyens (principes, réglementation, hiérarchisation...) qui leur sont favorables. Ils

\footnotetext{
${ }^{4}$ Les entreprises réseaux, virtuelles, les nouvelles formes d'organisations telles que le crowdsourcing illustrent ces évolutions de l'organisation.

${ }^{5}$ Par exemple, l'élu analysera une situation sous un angle politique, le technicien sous un angle technique.
} 
utilisent leur marge de liberté afin d'acquérir du pouvoir et d'infléchir le fonctionnement de l'organisation dans le sens de leurs intérêts en contribuant à l'énonciation des principes et des règles. Ces dernières sont la preuve de l'existence de relations de pouvoir, elles émergent de la structure formelle de l'organisation ou des pratiques informelles des acteurs.

Chaque acteur va chercher à jouer son propre jeu dans le cadre du SAC (Crozier, Friedberg, 1977) et à affirmer son pouvoir. Ce dernier dont les origines sont multiples ${ }^{6}$ se définit comme une relation asymétrique, un rapport de force. II exprime une relation d'échange, une négociation dont le résultat n'est pas fixé. Les principes et règles qui gouvernent le fonctionnement de l'organisation constituent à la fois des contraintes et des « espaces » ou les relations de pouvoir se développent. Ils génèrent des zones $d^{\prime}$ incertitude ${ }^{7}$ que les acteurs tentent de contrôler et d'utiliser pour servir leurs stratégies. Le contrôle de ces " espaces" passe par la maitrise de ressources qui offre des possibilités d'action et donc un espace de liberté (Crozier, Friedberg, 1977).

Ainsi, pour les auteurs de l'analyse stratégique en dehors de toute dimension hiérarchique, le pouvoir n'est pas lié aux personnes, mais aux ressources dont elles disposent. Selon cette logique instrumentale, l'acteur va utiliser ses ressources afin de développer son pouvoir.

Crozier et Friedberg font état de quatre ressources principales sources de pouvoir :

- la compétence,

- la maitrise de l'information sur l'environnement,

- le contrôle de la communication et de l'information interne,

\footnotetext{
${ }^{6}$ Les origines du pouvoir sont multiples: savoir, expertise, statut, légitimité...

7 L'incertitude peut provenir de failles dans les règles de fonctionnement, de pressions économiques et de contraintes issues de l'environnement, de défaillances techniques, de changements d'individus.
}

- la connaissance précise des règles de fonctionnement de l'organisation.

Si le cadre d'analyse proposé par Crozier et Friedberg permet de comprendre le fonctionnement des organisations, à partir des rapports de pouvoir qui les structurent et qui rendent les comportements des acteurs intelligibles, il ne fournit pas d'éléments quant à la façon d'identifier les acteurs concernés. Pour Friedberg (1993), la sociologie de l'action organisée peut être appliquée à toutes les formes d'action " organisée ", son domaine de prédilection reste les organisations à savoir des ensembles assez fortement codifiés, assez précisément délimités, où les acteurs sont durablement ensemble et où il existe un objectif - celui de l'organisation - partiellement différenciable des objectifs de chaque acteur. L'observation préalable (évoquée précédemment) des marques territoire nous a permis de mettre en évidence pour ces objets des organisations encore peu stabilisées (arrivée de nouveaux acteurs, désengagement de certains), peu codifiées et dont les règles et les principes de fonctionnement apparaissent très souples même si toutes disposent d'un code de marque (indice d'une démarche de codification). Lorsque les frontières de l'organisation se révèlent floues, le repérage des acteurs apparait délicat dans la mesure où l'action n'est pas toujours apparente. La théorie des parties prenantes (Freeman, 1984) en fournissant une approche étendue des ayants droits facilite ce repérage.

\subsection{La TPP pour une approche élargie de l'organisation}

La théorie des parties prenantes (TPP) à laquelle Freeman dans les années 80 a donné toute sa portée est largement mobilisée par les sciences de gestion. Son principe repose sur la nécessité pour les managers de ne pas uniquement focaliser leur attention sur les seuls actionnaires, mais d'intégrer dans les réflexions, les stratégies et les actions, le point de vue des autres ayants droits susceptibles d'affecter ou d'être affectés par les actions de l'entreprise (Freeman, 1984). Si la théorie a été essentiellement mobilisée 
pour analyser et pour étudier les relations d'entretiennent les entreprises et les individus ou groupes d'individus, elle présente un potentiel pour explorer les relations d'autres formes d'organisations avec les parties prenantes. Elle avance l'idée que l'organisation (l'entreprise dans la plupart des cas) doit travailler à équilibrer les intérêts concurrents des différents participants afin de maintenir la coopération nécessaire à son bon fonctionnement et à sa pérennité. Selon les recherches, la théorie des parties prenantes est mobilisée dans sa dimension descriptive, instrumentale, ou normative. Pour Donaldson et Preston (1995), elle est aussi managériale et stratégique, car elle débouche sur la recommandation d'attitudes, la mise en œuvre de structures et de pratiques qui constituent le management des parties prenantes. Dans sa dimension descriptive ou empirique, cette approche se concentre sur le comportement actuel de l'organisation. Elle cherche à décrire et expliquer comment l'organisation interagit avec les parties prenantes, cependant elle n'intègre pas la façon dont les parties prenantes interagissent entre elles: coordonnent leurs actions, régulent leurs relations, des aspects traités dans le cadre croziérien. Pour les décrire, les chercheurs se sont attachés à classer les parties prenantes. Diverses typologies ont été proposées. Les critères les plus fréquemment retenus sont la nature des parties prenantes (interne/externe ${ }^{8}$ ), les enjeux qui leurs sont associés (économiques ou sociaux, matériels ou symboliques), leur contribution (ressources tangibles ou intangibles), les relations contractuelles ou non contractuelles qu'elles entretiennent entre elles. Par exemple, Clarkson (1995) les classes selon le lien financier qu'elles cultivent avec l'entreprise. Ce lien détermine les obligations prioritaires de l'entreprise envers les parties prenantes primaires ou secondaires. La proximité est aussi couramment retenue.

\footnotetext{
${ }^{8}$ La classification interne ou externe des parties prenantes dans certaines organisations aux contours flous se révèle problématique. 96
}

L'identification des parties prenantes prioritaires est généralement réalisée à, partir de trois caractéristiques: le pouvoir, la légitimité et l'urgence (Mitchell, Agle et Wood, 1997). La première caractéristique, le pouvoir, peut être définie comme la capacité à conduire un acteur à réaliser quelque chose qu'il n'aurait pas fait de lui-même (Dahl, 1957), à influencer les décisions. II peut être coercitif, utilitariste ou normatif (Mitchell et al, 1997), et renvoie à l'exercice de pressions sociales, politiques ou fonctionnelles (Oliver, 1997). Il est associé aux ressources détenues et indispensables au fonctionnement de l'organisation et à sa stratégie et s'approche de la définition qu'en donnent Crozier et Friedberg (1977). II peut être acquis par le biais de relations développées avec d'autres parties prenantes. Son identification n'est pas toujours aisée. La seconde caractéristique, la légitimité, renvoie à un système socialement construit de normes, de valeurs dictant un comportement et des activités désirables et appropriées (Suchman, 1995). La troisième caractéristique, I'urgence, est associée aux contraintes de temps et à la gravité, c'est-àdire à l'importance de la demande exprimée par les parties prenantes. Sur la base de ces trois critères, Mitchell, Agle et Wood (1997) dégagent sept types de parties prenantes selon que la partie prenante présente un, deux ou trois des critères énoncés.

La dimension instrumentale avancée par Jones (1995) fournit un cadre pour examiner les relations entre le management (qui inclut les processus, structures et pratiques liés aux parties prenantes) et les objectifs de l'organisation telles que la rentabilité et la croissance (Donaldson, Preston, 1995). L'idée sous-jacente est que le management par les parties prenantes permet d'obtenir un avantage compétitif et une performance financière supérieure. Clarkson (1995) affirme que la performance et la survie de l'organisation dépendent de la capacité de ses managers à créer suffisamment de satisfaction et de valeur pour les parties prenantes. Enfin, la dimension normative / prescriptive est centrée sur la prescription de 
comportements issus de principes éthiques et moraux (Evan, Freeman, 1988). Cette approche est l'objet de débats dans les milieux académiques quant à son fondement. Elle indique la manière dont les organisations devraient traiter les parties prenantes. Selon cette approche, l'organisation doit porter une attention à toutes les demandes des parties prenantes et pas seulement à certaines d'entre elles.

La théorie des parties prenantes offre l'avantage d'offrir un cadre pour recenser les individus et groupes d'individus affectés ou pouvant affecter les actions de l'organisation ainsi que leurs motivations et moyens de pression et donc de pouvoir disposer d'une cartographie des parties prenantes utile au manager. Parmi les critiques couramment adressées à cette théorie, on relèvera plus particulièrement la diversité des cadres, méthodologies et perspectives mobilisés, la dimension réductrice du découpage en "parties prenantes" parfois très abstraites d'un ensemble d'acteurs sociaux avec des logiques complexes et diversifiées, le fait qu'elle n'appréhende pas vraiment la dynamique du processus de construction sociale et cognitive. Des limites que la théorie des acteurs de Crozier et Friedberg (1977) permet de dépasser. Ces deux cadres d'analyse apparaissent comme très complémentaires : la TPP permet d'identifier les parties prenantes touchées et/ou impliquées et la théorie des acteurs fournit les outils pour explorer les interactions, et comprendre la place occupée par chacune.

\section{Cadre d'analyse et méthodologique}

\subsection{L'objectif de la recherche}

La marque territoire résulte d'une action collective, elle est donc un phénomène social pouvant être analysé comme le produit des comportements d'un ensemble d'acteurs liés entre eux par de l'interdépendance stratégique et dont les actions renvoient les unes aux autres et forment un système qui obéit à un ordre "local » (Friedberg, 1993). Elle conduit à la mobilisation et la participation de nombreuses parties prenantes. Aussi, notre projet d'étudier ce phénomène nécessite d'identifier les acteurs impliqués, la façon dont ils interviennent dans le processus, les enjeux qu'ils mettent dans leurs actions, les ressources dont ils disposent et ce qu'ils attendent de leur participation à la marque. Dans cette perspective nous adoptons le raisonnement stratégique croziérien en nous centrant sur l'acteur et non pas sur le système. Nous ne nous intéressons pas à la façon dont se construisent les règles de fonctionnement de l'organisation, mais à la manière dont les acteurs conçoivent leur action. La perspective de cette recherche est donc phénoménologique et la logique retenue hypothético-inductive est destinée à fournir un modèle descriptif, voire interprétatif des données. Le point de départ de notre travail est l'acteur et sa stratégie.

\subsection{Le cas de la marque territoire Auvergne Nouveau Monde}

L'étude des acteurs prenant part à l'organisation d'une marque territoriale a été réalisée sur la marque de la région Auvergne sur la période 2013-2014. L'Auvergne compte parmi ces territoires français méconnus ou mal connus qui conservent une image archaïque, vieillissante et véhiculent de nombreux a priori. Ce déficit d'image dont souffre le territoire Auvergne est assez largement partagé par le grand public français, comme l'a montré l'enquête IPSOS réalisée par l'association Auvergne Nouveau Monde en $2012^{9}$. L'Auvergne, onzième région française en termes d'attractivité, de ce fait, cherche à accroître celle-ci en améliorant sa visibilité au-delà des frontières nationales au moyen d'une marque région. La marque «Auvergne Nouveau Monde» a été créée en septembre 2011 à l'initiative du conseil régional d'Auvergne et de ses quatre agences

\footnotetext{
9 Une étude IPSOS réalisée en 2012 sur un échantillon de 1500 personnes habitant en France hors Auvergne met en évidence un décalage entre l'image que les non auvergnats ont de l'Auvergne et la réalité.
} 
de développement territoriales rejoints par de grandes entreprises régionales, des PME, des festivals, des lieux culturels, des associations, des universités, etc., pour atteindre en 2014, environ 700 adhérents à l'association chargée de développer la marque. Portée sous forme associative, cette marque repose sur une volonté politique d'instaurer une démarche de marque «partagée» - entre habitants, collectivités, institutionnels, entreprises, et universités fondée sur une approche collaborative (coconstruite) de la marque (Rainisto, 2003 ; Kau, 2006). La marque territoire Auvergne Nouveau Monde (ANM) a suivi un processus de construction discontinu hétérogène (Rochette, Chanut, 2012). En reconstituant la chronologie, quelques étapes peuvent être distinguées comme le précise la figure 1 .

Figure 1. Historique de la marque Auvergne Nouveau Monde

\begin{tabular}{|c|c|c|c|c|c|}
\hline Fin 20082009 & 2010 & 2011 & 2012 & 2013 & $\begin{array}{l}\text { 1er semestre } \\
2014\end{array}$ \\
\hline $\begin{array}{l}\text { Lancement } \\
\text { d'une étude } \\
\text { d'identité } \\
\text { sur l'Auvergne } \\
\text { par le CRDT }\end{array}$ & $\begin{array}{l}\text { Septembre : } \\
\text { Projet de } \\
\text { marque }\end{array}$ & $\begin{array}{l}\text { Lancement } \\
\text { de la marque } \\
\text { Avril } 2011 \text { : création } \\
\text { de l'association } \\
\frac{\text { Juin } 2011}{\text { Communication }} \\
\text { officielle } \\
\text { Octobre } 2011 \text { : } \\
\text { Lancement officiel }\end{array}$ & $\begin{array}{l}\text { Recrutement } \\
\text { d'adhérents : } \\
350 \text { adhérents } \\
\text { Affinement du } \\
\text { positionnement } \\
\text { et du code de } \\
\text { marque }\end{array}$ & $\begin{array}{l}\text { Lancement de la } \\
\text { lère campagne } \\
\text { d'images et de } \\
\text { preuves } \\
\text { partagée ( } 7 \\
\text { partenaires } \\
\text { publics/privés } \\
\text { Lancement d'une } \\
\text { campagne } \\
\text { d'ambassadeurs } \\
\text { de marque }\end{array}$ & $\begin{array}{l}\text { Démarche de co- } \\
\text { construction des } \\
\text { outils de } \\
\text { communication } \\
\text { avec les } \\
\text { habitants (comité } \\
\text { pionnier, pépites) }\end{array}$ \\
\hline
\end{tabular}

Source : Les auteurs

Il convient de préciser que la marque Auvergne Nouveau Monde présente une importante singularité dans sa démarche. Celle-ci réside en premier lieu dans son mode de gouvernance, puisqu'elle est la seule marque dans le tissu des marques territoires françaises à être rattachée à une association sans être la propriété de la collectivité tout en rassemblant des acteurs privés et publics, institutionnels, économiques ou universitaires. Ce mode de gouvernance implique toutes les parties prenantes et l'équipe opérationnelle afin que les objectifs et les moyens de la démarche alloués aux différentes actions puissent être connus par l'ensemble des parties prenantes et pour permettre une efficience collective sur les projets. Présidée par un entrepreneur, la marque est pilotée par la vie associative que constituent les bureaux, les conseils d'administration et assemblées générales. Les membres du conseil d'administration, élus pour une durée de trois ans se réunissent trimestriellement afin de réaffirmer les grandes orientations de la marque. Le bureau, concentré autour des représentants de chaque catégorie de parties prenantes du conseil d'administration se réunit tous les mois afin de valider les points opérationnels et de mettre en œuvre le plan d'actions. Enfin, de manière statutaire, l'ensemble des adhérents se réunit une fois dans l'année pour procéder aux votes des rapports moraux et financiers de l'association et pour assister à la présentation du plan d'actions de l'année à venir. L'équipe opérationnelle est composée d'un directeur délégué chargé du développement de l'association et de deux chargés de mission travaillant à l'animation et à la mise en œuvre des actions. Cette équipe propose et met en œuvre les actions définies par le bureau et le conseil d'administration. La figure 2 reprend l'écosystème de la 
marque Auvergne Nouveau Monde et ses parties prenantes.

Figure 2. Écosystème de la marque Auvergne Nouveau Monde

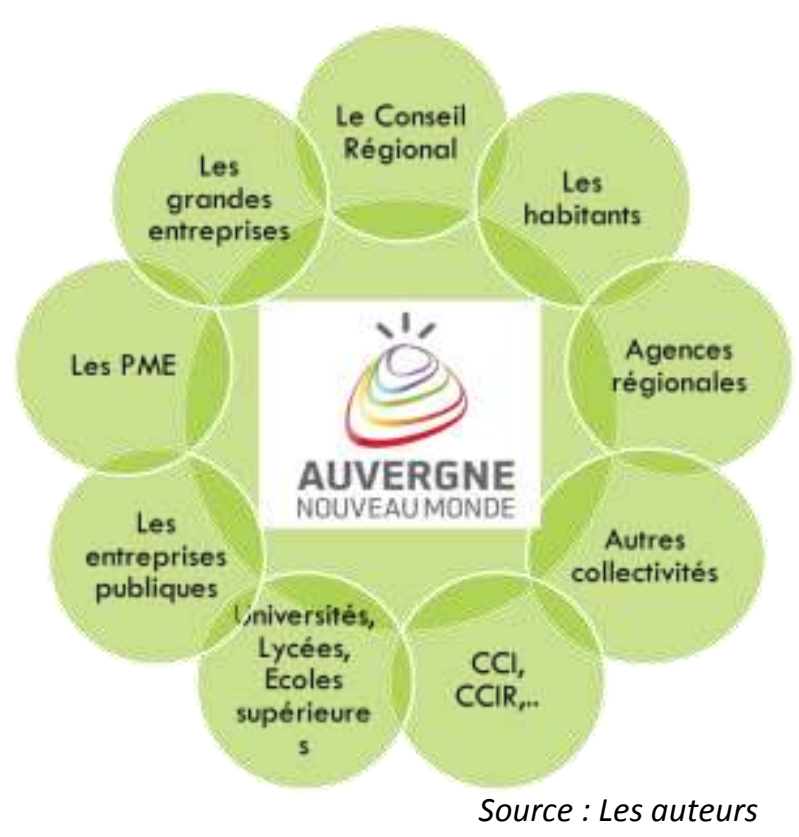

\subsection{L'approche mobilisée}

méthodologique

La méthodologie mobilisée pour étudier ce cas est celle de l'observation et de la recherche intervention. Un des chercheurs agit au sein de l'association Auvergne Nouveau Monde (ANM) dans le cadre d'une convention industrielle de formation par la recherche (CIFRE). Celui-ci occupe un poste de chargé de mission marketing créé un an après le lancement de l'association au sein d'une équipe restreinte ( 2 personnes la composent). Sa mission principale est la conception et la mise en œuvre d'outils marketing. Cette position facilite l'accès à des données variées et nombreuses qui dans un souci de rigueur sont scrupuleusement enregistrées dans des fiches.

\subsubsection{Une étude de cas dans le cadre d'une recherche-intervention}

Cette recherche à visée exploratoire (Yin, 1994) a pour terrain les différentes agences régionales de la région Auvergne: I'Association Auvergne Nouveau Monde, l'Agence Régionale de Développement Économique Auvergne, l'Agence Régionale de
Développement des Territoires d'Auvergne, et le Comité Régional de Développement Touristique d'Auvergne. Les missions confiées au chercheur au sein de l'association Auvergne Nouveau Monde consistent, en une collaboration avec l'ensemble des acteurs des agences de la région Auvergne afin de développer et mettre en œuvre des méthodes, outils et actions de marketing (plan marketing long terme et annuel, outils de reporting intégrant la création d'indicateurs de suivi, outils de veille, un système de collecte d'informations, mise en œuvre d'actions évènementielles).

\subsubsection{Le protocole de collecte et d'analyse des données}

L'observation directe des acteurs et l'analyse de documents internes et externes ont été effectuées durant les années 2013 et 2014, permettant de combiner plusieurs sources des données. La collecte des données s'est organisée en trois temps :

- Le premier a été consacré à une collecte et une analyse fouillée de données secondaires (articles de presse, sites internet, analyse des procès-verbaux du conseil d'administration de l'association ANM) afin d'identifier les parties prenantes au projet d'en dégager les grandes catégories et ainsi identifier les acteurs en présence ${ }^{8}$ (Rochette, Zumbo-Lebrument, 2014) ;

- Le second a permis dans le cadre d'un contrat CIFRE au sein de l'association Auvergne Nouveau Monde d'échanger aisément avec les acteurs eux-mêmes, de recueillir leur discours chemin faisant et d'en consigner le contenu dans un journal de bord ;

- Le troisième a consisté en une collecte et une analyse des fiches d'adhésion des 350 adhérents qui

8 Cette première étape a donné lieu à une communication en 2014 au congrès de l'Association française du marketing sur l'identification des parties prenantes. 


\section{Identification des enjeux dans la construction d'une marque région Corinne ROCHETTE, Cédrine ZUMBO-LEBRUMENT, Pierre FÉNIÈS}

devaient rédiger un petit texte sur les raisons qui les poussaient à rejoindre l'association porteuse de la marque, permettant ainsi de connaître leurs objectifs et leurs motivations.

Les données collectées étaient destinées à repérer les variables clés qui constituent des éléments de l'analyse stratégique et de la théorie des parties prenantes. La grille de codage des données (tableau 2) a été structurée autour des notions de " objectifs » (nous cherchions à identifier ceux manifestes de ceux latents), "sources de pouvoir » (reprenant les quatre catégories énoncées précédemment) et "stratégies » pour les critères relatifs à l'approche croziérienne et complétée par les notions d' " attentes et intérêt ", de "ressources mobilisables " et " moyens d'actions et de pression ».

Tableau 2 : Grille de codage des données (source : les auteurs)

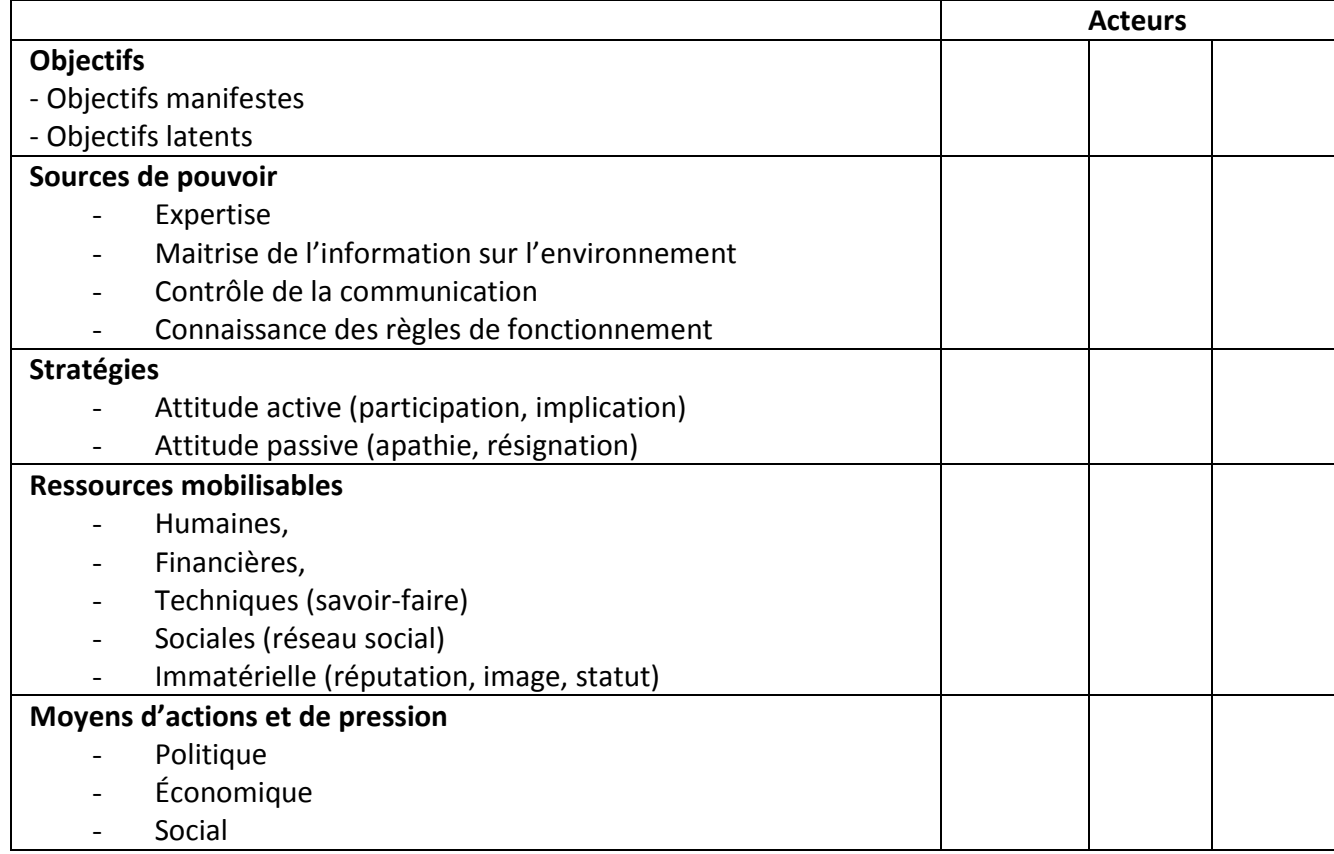

\section{Résultats et discussion de l'analyse des stratégies développées par les acteurs}

\subsection{Les résultats clés}

La mise en place d'une marque territoire partagée se heurte à des comportements parfois paradoxaux de la part des acteurs qui y participent. Leurs comportements peuvent freiner la mise en place de la démarche de la marque et constituent à ce titre un élément sensible, aussi, par mesure de confidentialité, les résultats seront représentés par grandes catégories d'acteurs (grandes entreprises, PME, collectivités, organismes consulaires...). La grille de codage appliquée à chaque catégorie est synthétisée sous forme de tableaux reprenant l'identification des éléments de positionnement des acteurs envers la marque ANM. Les analyses qui en sont faites sont destinées à mieux qualifier la nature et l'importance de la relation qu'entretient l'acteur avec l'association Auvergne Nouveau Monde. 
Tableau 3 : Identification des éléments du positionnement du Conseil Régional

\begin{tabular}{|l|l|}
\hline \multicolumn{2}{|c|}{ Conseil Régional d'Auvergne } \\
\hline Objectifs & - Améliorer l'attractivité de la région \\
- Objectifs manifestes & - Accroitre la notoriété de la région \\
& - Mobiliser sur un projet collectif \\
- Objectifs latents & - Homogénéiser les prises de parole \\
& - Utiliser la marque comme un instrument politique \\
\hline Sources de pouvoir & - Expertise (budgétaire) \\
& - Maitrise de l'information sur l'environnement \\
\hline Stratégies & - Connaissance des règles de fonctionnement \\
\hline Ressources mobilisables & - Attitude active (participation, implication) \\
\hline Moyens d'actions et de pression & - Financières \\
& - Techniques (savoir-faire) \\
\hline
\end{tabular}

Le conseil régional est l'instigateur du projet de la marque Auvergne Nouveau Monde via son président, la relation qu'il entretient avec I'association ANM est importante et même déterminante. II a imaginé la marque comme un outil de promotion du territoire et de mobilisation des énergies autour d'un projet commun, celui de changer l'image de l'Auvergne. À travers une occupation plus importante de l'espace médiatique sur la scène locale et nationale via sa marque territoire, la collectivité entend probablement affirmer indirectement son poids (objectif latent).

Malgré son autonomie organisationnelle et décisionnelle touchant à la mise en œuvre et la vie courante de la marque ANM, l'association ANM est dépendante des sources de pouvoir, essentiellement budgétaires, qui constituent l'une des principales zones d'incertitude. A celle-ci $s^{\prime}$ ajoute désormais celle issue de la réforme territoriale et du devenir des structures.

Tableau 4 : Identification des éléments du positionnement des agences régionales

\begin{tabular}{|l|l|}
\hline \multicolumn{2}{|c|}{ Agences régionales de développement } \\
\hline - Objectifs & $\begin{array}{l}\text { - Contribuer à la réalisation de leurs missions (attractivité économique, } \\
\text { touristique, accueil de nouvelles populations, évènements culturels) en } \\
\text { développant une marque co-construite }\end{array}$ \\
- Objectifs latents & $\begin{array}{l}\text { - Renforcer l'adhésion des parties prenantes } \\
\text { - Mutualisation des budgets et des actions }\end{array}$ \\
& - Agir sur la cohérence des actions \\
& - Acquérir des outils marketing \\
\hline Sources de pouvoir & - Expertise budgétaire \\
& - Maitrise de l'information sur l'environnement \\
& - Contrôle de la communication \\
& - Connaissance des règles de fonctionnement \\
\hline Stratégies & - Attitude active (participation, implication) \\
\hline Ressources mobilisables & - Humaines \\
& - Financières \\
\hline Moyens d'actions et de pression & - Sociales (réseau) \\
\hline
\end{tabular}

Les agences de développement du conseil régional sont, à n'en pas douter, des acteurs cardinaux dans le dispositif de la marque
ANM. En effet, la valeur de la relation avec ces dernières est forte au regard des zones d'incertitudes sur lesquelles elles possèdent 


\section{Identification des enjeux dans la construction d'une marque région Corinne ROCHETTE, Cédrine ZUMBO-LEBRUMENT, Pierre FÉNIÈS}

des marges de manœuvre. Leurs compétences en matière de communication, leur volonté d'allouer des ressources financières à ANM ainsi que leur capacité à posséder et à diffuser certaines informations par le biais de leurs réseaux relationnels font de leur implication un enjeu déterminant du développement de la marque. Par ailleurs, ces

Tableau 5 : Identification des éléments du positionnement des collectivités autres que le conseil régional

\begin{tabular}{|l|l|}
\hline \multicolumn{2}{|c|}{ Département et autres collectivités territoriales } \\
\hline $\begin{array}{l}\text { Objectifs } \\
\text { - Objectifs manifestes }\end{array}$ & $\begin{array}{l}\text { - Améliorer l'attractivité de leur territoire } \\
\text { - Mobiliser leur territoire sur un projet collectif }\end{array}$ \\
& $\begin{array}{l}\text { - Bénéficier d'une notoriété (région) plus forte et de retombées (image, } \\
\text { attractivité) } \\
\text { - Accroître leur visibilité } \\
\text { - Mutualiser les moyens financiers }\end{array}$ \\
\hline Sources de pouvoir & - Maitrise de l'information sur l'environnement \\
\hline Stratégies & - Attitude neutre \\
\hline Ressources mobilisables & - Financières, \\
& - Sociales (réseau) \\
\hline Moyens d'actions et de pression & - Politique \\
& - Social \\
\hline
\end{tabular}

La valeur de la relation avec les départements peut être estimée comme faible pour I'association ANM. En effet, dans le cadre du système d'acteurs qu'est le projet de la marque ANM, les départements ne contrôlent qu'une source de pouvoir, celle inhérente à la maîtrise de leur environnement. II est lié à leur espace d'intervention. Des collectivités territoriales présentes sur le territoire auvergnat ont développé leur propre marque en l'ancrant dans la proximité (avec leurs administrés), aussi leur attitude envers ANM peut être qualifiée de plutôt passive. liens étroits avec ANM contribuent à légitimer leur existence et de leur mission.

Bien qu'étant de l'initiative d'une institution régionale, la marque ANM incarne un projet de territoire. Dans son message elle englobe les territoires plus restreints (villes, départements).
Néanmoins, certaines contribuent financièrement à ANM et diffusent la marque régionale en l'associant à la leur. A terme la recherche de synergies, l'alignement des pratiques, la rationalisation liée au contexte devraient permettre une incorporation plus forte et ainsi limiter cette zone d'incertitude.

L'objectif de l'association porteuse de la marque est de contribuer au renforcement de l'attractivité du territoire sur une cible jugée déterminante pour l'avenir du territoire celle des jeunes.

Tableau 6 : Identification des éléments du positionnement de l'éducation et de la recherche

\begin{tabular}{|l|l|}
\hline \multicolumn{2}{|c|}{ Acteurs de la sphère éducative et de la recherche } \\
\hline - Objectifs & $\begin{array}{l}\text { - Améliorer l'image de la région auprès de leurs cibles : étudiants auvergnats et } \\
\text { d'autres régions }\end{array}$ \\
- Objectifs latents & $\begin{array}{l}\text { - Valoriser leurs actions } \\
\text { - Bénéficier de moyens supplémentaires }\end{array}$ \\
\hline Sources de pouvoir & - Maitrise de l'information sur environnement \\
\hline Stratégies & - Attitude neutre \\
\hline Ressources mobilisables & - Sociales (réseau social) \\
\hline Moyens d'actions et de pression & - Immatérielle (réputation, image, statut) \\
\hline
\end{tabular}


Les acteurs de la sphère éducative et de la recherche contrôlent une partie du lien entre I'association ANM et son environnement. Leur lien avec la jeunesse les rend incontournables. Ces jeunes constituent des ambassadeurs de la marque ANM, ils contribuent à diffuser l'image de la région, leur mode de communication très réticulaire permet d'accélérer le processus de diffusion, leur prise de parole de rajeunir l'image perçue. En ce sens, la relation avec les acteurs de la sphère éducative et de la recherche revêt un enjeu important pour l'association ANM de par la dimension sociale à laquelle ils sont associés.

En tant que marque partagée la marque ANM mobilise aussi la sphère privée et en particulier les entreprises.

Tableau 7 : Identification des éléments du positionnement des grandes entreprises

\begin{tabular}{|l|l|}
\hline \multicolumn{2}{|c|}{ Grandes entreprises } \\
\hline $\begin{array}{l}\text { Objectifs } \\
\text { - Objectifs manifestes }\end{array}$ & $\begin{array}{l}\text { - Participer à la construction d'une marque territoire visant à renforcer l'attractivité de } \\
\text { l'Auvergne }\end{array}$ \\
- Objectifs latents & $\begin{array}{l}\text { Affirmer leur appartenance territoriale (dimension politique et facteur de } \\
\text { différenciation/consommateur) } \\
\text { - Renforcer l'image d'entreprise citoyenne } \\
\text { - Attirer de nouveaux salariés }\end{array}$ \\
\hline Sources de pouvoir & - Expertise \\
& - Maitrise de l'information sur l'environnement \\
\hline Stratégies & - Attitude active (participation, implication) \\
\hline Ressources mobilisables & - Financières \\
& - Techniques (savoir-faire) \\
Moyens d'actions et de & - Immatérielle (réputation, image, statut) \\
\hline pression & - Économique \\
\hline
\end{tabular}

L'intensité, le contenu et donc la valeur de la relation entre les grandes entreprises et ANM peuvent être évalués comme moyenne, par référence aux sources de pouvoir. En effet, bien qu'existantes (expertise, maitrise de I'information sur l'environnement) ces sources de pouvoir ne conditionnent pas la survie de la marque et constituent des zones d'incertitude atténuées. Les grandes entreprises, du fait de leur déploiement national et international, représentent un vecteur d'informations sur les pratiques en marketing territorial d'autres régions; elles sont également un accélérateur d'accès à certaines ressources rares (compétences et financières); enfin, l'intégration de la RSE (responsabilité sociale de l'entreprise) leur a permis de développer des interfaces avec d'autres systèmes d'action concrets pouvant potentiellement bénéficier à la marque ANM. La situation est bien différente pour les entreprises de taille plus restreinte.

Tableau 8 : Identification des éléments du positionnement des PME

\begin{tabular}{|l|l|}
\hline \multicolumn{2}{|c|}{ PME } \\
\hline $\begin{array}{l}\text { Objectifs } \\
\text { - Objectifs manifestes }\end{array}$ & $\begin{array}{l}\text { Participer à la construction d'une marque territoire visant à renforcer l'attractivité de } \\
\text { I'Auvergne }\end{array}$ \\
- Objectifs latents & $\begin{array}{l}\text { - Retombées économiques } \\
\text { - Accroitre leur visibilité } \\
\text { - Attirer de nouveaux salariés }\end{array}$ \\
\hline Sources de pouvoir & - Maitrise de l'information sur l'environnement \\
\hline Stratégies & - Attitude neutre \\
\hline Ressources mobilisables & - Financières \\
& - Sociales (réseau social) \\
\hline $\begin{array}{l}\text { Moyens d'actions et de } \\
\text { pression }\end{array}$ & - Économique \\
\hline
\end{tabular}


Les PME (en tant qu'entité individuelle) entretiennent généralement des rapports étroits avec leur territoire; cependant, la faiblesse des ressources dont elles disposent habituellement, leur espace d'intervention plus restreint limitent naturellement leurs contributions. La valeur de la relation d'ANM avec les PME peut être estimée comme moyenne. La particularité par rapport aux grandes entreprises réside essentiellement dans la différence de nature du lien qu'ANM développe avec les $\mathrm{PME}$, un lien qui s'inscrit dans la proximité géographique et qui est plus destiné à créer une dynamique locale, encourager d'autres PME à adhérer au projet et dans la mesure du possible à contribuer financièrement. II s'agit donc de susciter leur intérêt pour une question (la marque ANM) qui n'apparait pas comme un enjeu. Les PME se caractérisent aussi par l'importance des relations qu'elles développent entre elles, par leur appartenance à des réseaux socioéconomiques pouvant être appréhendés comme d'éventuels leviers de mobilisation.

Tableau 9 : Identification des éléments du positionnement des consultants

\begin{tabular}{|l|l|}
\hline \multicolumn{2}{|c|}{ Consultants } \\
\hline $\begin{array}{l}\text { Objectifs } \\
\text { - Objectifs manifestes }\end{array}$ & $\begin{array}{l}\text { Participer à la construction d'une marque territoire visant à renforcer l'attractivité } \\
\text { de l'Auvergne }\end{array}$ \\
- Objectifs latents & $\begin{array}{l}\text { - Vendre leurs prestations } \\
\text { - Devenir une référence en matière de marketing territorial }\end{array}$ \\
\hline Sources de pouvoir & - Expertise \\
\hline Stratégies & - Attitude neutre \\
\hline Ressources mobilisables & $\begin{array}{l}\text { - Techniques (savoir-faire) } \\
\text { - Immatérielle (réputation, image, statut) }\end{array}$ \\
\hline Moyens d'actions et de pression & Non concernés \\
\hline
\end{tabular}

L'association ANM développe une relation avec les consultants pour avoir accès à la ressource que sont les compétences techniques relatives au marketing territorial. Cette ressource était, au départ, déterminante, puisque la structure porteuse du projet de marque territoire ne disposait pas de compétences de nature marketing, elle n'avait pas connaissance de la démarche à adopter et elle ne maitrisait donc pas le processus de création: la compétence (expertise en marketing territorial) était donc source d'un enjeu important. Par ailleurs, pour les consultants, l'accompagnement des régions contribue à renforcer leur crédibilité et leur capacité à vendre leurs services à d'autres organisations. Le poids des consultants s'est estompé avec le temps et l'acquisition d'un savoir-faire par les porteurs du projet de la marque ANM.

Tableau 10 : Identification des éléments du positionnement des habitants

\begin{tabular}{|l|l|}
\hline \multicolumn{2}{|c|}{ Habitants } \\
\hline $\begin{array}{l}\text { Objectifs } \\
\text { - Objectifs manifestes }\end{array}$ & - Renforcer le sentiment d'appartenance à leur territoire \\
- Objectifs latents & - Accroître leur capital social \\
\hline Sources de pouvoir & - Maitrise de l'information sur l'environnement \\
& - Compétences (mais atténuée cf créativité) \\
\hline Stratégies & - Attitude active (participation, implication) \\
\hline Ressources mobilisables & - Sociales (réseau social) \\
\hline Moyens d'actions et de pression & - Immatérielle (réputation, image, statut) \\
\hline
\end{tabular}

Les habitants sont des acteurs du projet de marque de territoire dont la valeur de la relation avec l'association ANM est importante dans le cadre d'une marque régionale partagée et co-construite. En effet, dans le cadre d'une marque partagée, les habitants sont, de fait, tous porteurs d'une partie du lien de la marque ANM avec son environnement. Par ailleurs, certaines démarches de marketing visant à la co- 
construction de la marque sont menées (Les pionniers) en faisant appel à des compétences possédées par des habitants. Ces deux sources d'incertitude pour l'association ANM font de la relation avec les habitants un enjeu déterminant à court, moyen et long terme.

Les autres acteurs (entreprises publiques, chambres consulaires, marques collectives agro-alimentaires) sont, considérés du point de vue de l'association ANM, comme porteurs d'un enjeu faible ou moyen dans la mesure où ils peuvent être analysés comme détenteurs d'une unique source de pouvoir : la maîtrise de l'information pour les deux dernières catégories d'acteurs: organisations consulaires et les marques agroalimentaires. Globalement, leur attitude face à la marque est plutôt passive. Elles ne mobilisent pas de ressources particulières pour la marque territoire en dehors de leur réseau social. Si pour les organisations consulaires les moyens d'action sont politiques et sociaux, ils sont plutôt économiques pour les marques agroalimentaires.

\subsection{Quelques éléments de discussion et pistes d'actions}

Les grilles synthétiques présentées permettent de dégager les éléments pouvant expliquer les jeux d'acteurs et leur attitude envers la marque entre engagement et retrait, autour des cinq grandes rubriques utilisées dans la grille de lecture :

- On constate d'abord des différences entre les objectifs manifestes et ceux latents, cependant ceux-ci ne s'excluent pas. Ils peuvent coexister sans engendrer de tensions.

- Les sources de pouvoir se situent d'abord dans la maitrise de l'information sur l'environnement dont disposent les acteurs et ensuite dans l'expertise. Les acteurs constituent des relais pour asseoir la marque et l'aider à trouver sa place. Ils sont les principaux pourvoyeurs d'informations sur les domaines qu'ils couvrent. En partageant cette information avec ANM, ils lui apportent un éclairage sur les modes de fonctionnement $\mathrm{du}$ domaine, les évolutions, défis et les préoccupations, et ainsi permettent à la marque de développer des actions plus en phase avec les attentes des acteurs. Par exemple, l'information dont disposent les universités sur les étudiants (leurs origines géographiques, les filières plébiscitées), mais aussi sur l'attente des entreprises en matière de formation des futurs diplômés peut permettre de mieux cibler les actions de l'association (présence sur des salons nationaux ou internationaux, concours pour des aides à I'initiative étudiante sur un secteur particulier). Aussi, il apparait important de prévoir des moments d'échange (entre acteurs publics, mais aussi publics et privés) pour faciliter la connaissance pour les porteurs des marques territoire du fonctionnement et des évolutions sectoriels. Une deuxième source de pouvoir apparait nettement; l'expertise. Sur un plan technique (marketing territorial), celle-ci a été acquise par le recours à des consultants, I'univers public disposant globalement de peu de compétences en matière de pilotage de projets marketing. Les compétences " marketing " des habitants (créativité, imagination) même si elles s'expriment timidement (via les comités pionniers) existent et constituent une forme d'expertise. Pour les grandes entreprises qui disposent de compétences dans ce domaine, le pouvoir ne se situe pourtant pas dans cette expertise probablement en raison des spécificités du marketing territorial, qui renvoie à des approches très aménagées du marketing classique.

- L'attitude des acteurs est très variable. Assez logiquement, les organisations publiques régionales ont une attitude très active, alors qu'elles se révèlent plus passives pour celles agissant sur d'autres échelons territoriaux (communes, départements). La démarche est probablement perçue, par ces acteurs-là, comme centralisée (voire confisquée par 


\section{Identification des enjeux dans la construction d'une marque région Corinne ROCHETTE, Cédrine ZUMBO-LEBRUMENT, Pierre FÉNIÈS}

la région) plus que partagée. II convient de noter la fréquence des attitudes neutres, ANM doit travailler à susciter l'intérêt pour réduire cette forme d'indifférence au projet.

- Les ressources mobilisables par les acteurs sont majoritairement sociales (réseau). La ressource financière reste très associée aux structures publiques et celles immatérielles aux entreprises et associations agroalimentaires, les labels tels que les $A O C$ peuvent constituer de par leur image et statut une ressource à mobiliser.

- Quant aux moyens d'action, ils sont avant tout économiques. La dimension politique associée à ses marques territoire en fait par nature des marques quasi politiques destinées à affirmer des choix en termes de projet de territoire, les moyens d'action politique apparaissent aussi nettement.

Les tableaux présentés fournissent aux personnes en charge du déploiement de la marque une première grille de décodage des comportements dans le but d'ajuster les moyens d'action et construire des argumentaires en lien avec les objectifs et ressources dont dispose chacun de ces acteurs.

Une telle lecture réalisée très en amont du lancement $d$ 'une marque de territoire permettrait de développer des stratégies particulièrement adaptées et ainsi réduirait probablement la durée et l'intensité de la phase d'ajustement, de confrontation et dialogue nécessaire pour mettre en œuvre un projet partagé, mobilisateur et véritablement consensuel. Les résultats mettent en avant la nécessité de valoriser, par-delà une approche politique du projet de marque, l'importance des dimensions humaines et organisationnelles inhérentes au lancement et au pilotage d'une marque de territoire. En effet, un projet de marque de territoire ne peut se résumer à la conception et la mise en place d'un plan marketing et de communication qui, si elles sont des étapes nécessaires, ne peuvent être suffisantes pour pérenniser la marque région. Même si celle-ci repose d'abord et avant tout sur l'implication et l'engagement de tous les acteurs, parties prenantes du projet, cela suppose l'intégration au sein du projet d'une réelle démarche de conduite du changement fondée sur la connaissance et l'appréciation des caractéristiques inhérentes à chacun des acteurs-parties prenantes (motivations, objectifs, sources de pouvoirs, ressources mobilisables, stratégie prévisible).

\section{Conclusion}

La recherche sur le marketing territorial s'est concentrée sur ses dimensions communicationnelles et instrumentales (dont la marque) explorant peu la place des acteurs pourtant fondamentale dans un projet de territoire qui par essence est collectif. La théorie des acteurs et celle des parties prenantes offrent un cadre aisément mobilisable pour explorer les dynamiques sociales à l'œuvre dans un projet de marque territoire. C'est sur la base de ces deux cadres théoriques que nous avons proposé une grille de lecture pour identifier les éléments à l'origine du positionnement des acteurs par rapport au projet de marque. Cette grille a été appliquée au cas de la marque région Auvergne Nouveau Monde afin de pouvoir se représenter le positionnement des grandes catégories d'acteurs, de fournir aux managers publics des clés de lecture des comportements et de suggérer des pistes d'actions.

L'apport de cette recherche réside donc dans l'usage conjoint d'éléments de lecture issus de la théorie des parties prenantes et de l'analyse stratégique croziérienne afin $d^{\prime}$ 'identifier et d'estimer la nature, la valeur et les enjeux des différentes relations nouées par l'association ANM, porteuse la marque. Elle a ainsi permis de mieux délimiter les objectifs et les sources de pouvoir des acteurs participant du processus de mise en marque. La théorie des parties prenantes est utilisée avec pour dessein d'en exploiter la capacité d'identification et de description des acteurs ; l'analyse stratégique est employée pour sa 
vertu prescriptive afin de précisément estimer les relations, dégager les zones d'incertitudes et les enjeux associés au système d'action concret qu'incarnent la structure organisationnelle d'ANM.

\section{Bibliographie}

ANHOLT S., 2010, "Definitions of place branding: working towards a resolution", Place Branding and Public Diplomacy, 6, 1, p. 1-10.

BAYGERT N., 2014, "Le nouveau branding wallon », Outre-Terre, 40, 3, p. 156-165.

CHAMARD C. (ss dir.), 2014, "Le marketing territorial-Comment développer l'attractivité et l'hospitalité des territoires?", De Boeck, Bruxelles.

CHANUT V., ROCHETTE C., 2012, "La fabrique d'une marque Région : le cas de la marque " Auvergne Nouveau Monde " ", Politiques et Management Public, 29, 3, p. 493-517.

CLARKSON M.B., 1995, “A Stakeholder Framework for Analyzing and Evaluating Corporate Social Performance", Academy of Management Review, 20, 1, p. 92-117.

CROUCH G.I., RITCHIE J.B., 1999, "Tourism, competitiveness, and societal prosperity", Journal of business research, 44, 3, p. 137-152.

CROZIER M., FRIEDBERG E., 1977, L'acteur et le système : Les contraintes de l'action collective, Seuil, Paris.

CROZIER M., 1989, L'entreprise à l'écoute : apprendre le management post-industriel, InterEditions, Paris.

DAHL R.A., 1957, "The Concept of Power", Behavioral Science, 2, 3, p. 201-215.

DAVID A., 2000, " La recherche intervention, un cadre général pour les sciences de gestion ? ", IX Conférence Internationale de Management Stratégique, Montpellier.

DE CHERNATONY L., 1999, "Brand management through narrowing the gap between brand identity and brand reputation", Journal of Marketing Management, 15, 1-3, p. 157-179.

DI MEO G., 1996, Les territoires du quotidien, L'Harmattan, Paris.
DONALDSON T, PRESTON L.E., 1995, "The stakeholder Theory of the corporation: concepts, evidence and implications", Academy of Management Review, 20, 1, p. 65-91.

DUMONT G.F., 2013, "Lyon: la revanche d'une métropole? ", Population et Avenir, 712, 2, p. 3-3.

ESHUIS J., BRAUN E., KLIJN E.H., 2013, "Place Marketing as Governance Strategy: An Assessment of Obstacles in Place Marketing and Their Effects on Attracting Target Groups", Public Administration Review, 73, 3, p. 507-516.

EVAN W.M., FREEMAN R.E., 1988, "A Stakeholder Theory of the Modern Corporation: Kantian Capitalism", in BEAUCHAMP T.L., BOWIE N.E. (éd.), Ethical Theory and Business, Englewood Cliffs, Prentice Hall, N.J., p. 97-106.

FREEMAN R.E., 1984, Strategic Management: A Stakeholder Approach, Pitman, Boston.

FRIEDBERG E., 1993, Le pouvoir et la règle. Dynamiques de l'action organisée, Seuil, Paris.

GIRARD V., 1999, Contribution à l'étude du marketing territorial: le cas des projets de ville et d'agglomération françaises, Thèse de Doctorat, Université de Lyon 3.

GOLLAIN V., 2014, Réussir sa démarche de marketing territorial : Méthode, techniques et bonnes pratiques, Territorial Editions, Paris.

HATEM F., 2004, Investissement international et politiques d'attractivité, Economica, Paris.

JONES T.M., 1995, "Instrumental stakeholder theory: A synthesis of ethics and economics", Academy of Management Review, 20, 2, p. 404437.

HOULLIER-GUIBERT C.E., 2009, "Quelles limites territoriales pour une promotion métropolitaine? Le cas de Rennes", Revue internationale de Communication sociale et publique, 1, 1, p. 127144.

HOULLIER-GUIBERT C.E., 2011, « La fabrication de l'image officielle de la ville pour un rayonnement européen : gouvernance, idéologies, coopération territoriale et rayonnement ", Cahiers de géographie du Québec, 55, 154, p. 7-35

KAU E., 2006, "Regional Branding: A Relationship of Public and Private Sectors", UW-L Journal of Undergraduate Research, 9, p. 1-6.

KAVARATZIS M., 2004, "From city marketing to city branding: Towards a theoretical framework for 


\section{Identification des enjeux dans la construction d'une marque région Corinne ROCHETTE, Cédrine ZUMBO-LEBRUMENT, Pierre FÉNIÈS}

developing city brands", Place branding, 1, 1, p. 58-73.

MEYRONIN B., 2009, Le marketing Territorial: enjeux et pratiques, Vuibert, Paris.

MICHELET J.F., GIRAUT F., 2014, "Construction d'une qualité régionale. La marque Valais ou les vertus et les risques du branding territorial ", Revue de géographie alpine, 102-1, p. 1-13.

MITCHELL R.K., AGLE B.R., WOOD D.J., 1997, "Toward a theory of stakeholder identification and salience; defining the principle of who and what really counts", Academy of Management Review, 22,4 , pp. 853-886.

MULLENBACH-SERVAYRE A. (2007), L'apport des parties prenantes à la modélisation de la responsabilité sociale des entreprises, La Revue des Sciences de Gestion, n²23, p. 109-120.

MUSSON A., 2012, "Construire l'attractivité durable régionale: l'exemple de l'Aquitaine ", n¹202, Larefi Working Papers, Larefi, Université Bordeaux 4

NOISETTE P., VALLERUGO F., 1996, Le marketing des villes : un défi pour le développement stratégique, Editions. d'Organisation, Paris.

OLIVER C., 1997, "Sustainable competitive advantage: combining institutional and ressourcebased views", Stategic Management Journal, 18, 9, p. 697-713.

PROULX M.U., TREMBLAY D., 2006, « Marketing territorial et positionnement mondial ", Géographie, économie, société, 8, 2, p. 239-256.

RAINISTO S.K., 2003, Success factors of place marketing: A study of place marketing practices in Northern Europe and the United States, Dissertation for the degree of Doctor of Science in Technology, Helsinki University of Technology.

ROCHETTE C., 2014, ZUMBO-LEBRUMENT C. "L'identification des parties prenantes impliquées dans la construction d'une marque région " (Le cas de la marque Auvergne Nouveau Monde), Colloque AFM, mai, Montpellier, France.

SUCHMAN M.C., 1995, "Managing Legitimacy: Strategic and Institutional Approaches", Academy of Management Review, 20, 3, pp. 571-610.

TEXIER L., VALLA J.P., 1992, « Le marketing territorial et ses enjeux ", Revue Française de Gestion, 87, p 45-55.

THIARD P., 2007, "Attractivité et compétitivité: offre territoriale, approches marketing et retombées ", PUCA, L'attractivité des territoires: regards croisés, Actes des séminaires, Paris, p. 5153.

YIN R.K., 1994, Case study research: Design and methods, Sage publications, Thousand Oaks, Calif. 